\title{
¿Innovación imitativa?: ¡Viva la imitación!
}

La innovación tecnológica comúnmente se relaciona con la generación de productos nuevos y radicales, en donde predomina la $1+D$ y las estrategias de apropiabilidad. En cambio, las innovaciones incrementales surgen a partir de diseños establecidos y no tienen el poder de generar nuevos mercados. Bajo este escenario surge la imitación como un fenómeno complejo y controvertido. Normalmente se relaciona con adquirir ventajas competitivas de manera fácil y oportunista. El presente artículo, aborda el fenómeno de la imitación en la industria tecnológica y discute el rol de la novedad, la propiedad intelectual y sus implicancias en economías emergentes.

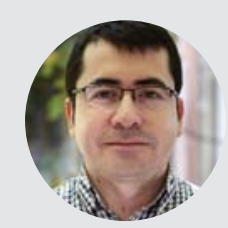

Alejandro Jiménez-Montecinos PROFESOR DE TECNOLOGÍA E INNOVACIÓN. DEPARTAMENTO DE GESTION Y NEGOCIOS, FEN-UAH

ljimenez@uahurtado.cl

\section{Un poco de historia}

Un aspecto difícil de aceptar para los teóricos de la innovación es la evidencia de que la mayoría de las innovaciones surgen por imitación. Una imitación que es propia de los homínidos y que ha permitido evitar los costosos errores de los pioneros en el desarrollo de la cultura humana (Velez, 2007).

En primera instancia, hay una aparente contradicción entre imitación e innovación, pues esta última se relaciona con la generación de algo nuevo y radicalmente distinto. En su definición clásica de desarrollo económico, Schumpeter (1934) habla de introducir productos o servicios que los consumidores aún desconocen, crear nuevos métodos de producción, abrir nuevos mercados o conquistar nuevas fuentes de suministros. 0 sea, debe existir un importante componente de novedad para que la innovación se constituya como tal. Bajo esa premisa, los imitadores quedan al margen de la definición por llegar en segundo lugar, tienen que adaptarse o, en el peor de los casos, se apropian de los esfuerzos del primero para adquirir una ventaja competitiva.

La literatura considera la imitación como algo opuesto a la innovación (Coad, 2011). El relato clásico es que los innovadores empujan la economía con sus nuevas tecnologías (innovaciones radicales o disruptivas). Mediante la investigación y desarrollo (l+D), los innovadores exploran la frontera del conocimiento lo que conlleva a pagar los costos del pionero. Sin embargo, son recompensados temporalmente por el monopolio legal que le otorgan sus estrategias de apropiabilidad (patentes y/o licencias de exclusividad). Además, la innovación radical tiene como consecuencia cierto grado de especialización, lo que obliga a las empresas a deshacerse de muchos activos para ser eficientes. Lo anterior suele conducir a lo que Christensen (2013) llama el dilema del innovador; empresas atrapadas en la mejora continua de su diseño original y que terminan incapaces de reconvertirse? Un planteamiento parecido sugiere March (1994), cuando se refiere a los llamados exploradores y explotadores. Los primeros se obsesionan con la novedad, el descubrimiento y la generación de productos constantemente; sin embargo, no tienen la capacidad de perseverar en el tiempo y son incapaces de posicionarse exitosamente en el mercado. Por otro lado, están los explotadores, que se vuelven especialistas en tomar el conocimiento existente y mejorarlo; desafortunadamente son tan buenos y eficientes que rechazan la posibilidad de cambio y terminan obsoletos ${ }^{2}$

Una vez que la innovación radical se establece, comienza la innovación incremental, que hace referencia a invenciones que introducen conceptos comunes y que pueden ser directamente derivadas de invenciones ya existentes. A diferencia

(1) En estricto rigor no es lo mismo innovación radical e innovación disruptiva. Aquí utilizo el término indistintamente para construir una analogía entre el primero que desarrolla la tecnología y el segundo que genera derivados y/o luego imita. Para una mejor precisión recomiendo leer el artículo: What is disruptive innovation? de Christensen y col. (2015) publicada en la Harvard Business Review https://hbr.org/2015/12/what-is-disruptive-innovation 
de la innovación radical, la innovación incremental no tiene el potencial para generar nuevos mercados. Pueden no proporcionar un beneficio superior para los clientes y están fuertemente influenciados por las invenciones pasadas. Los economistas sugieren que las empresas invertirán en grandes innovaciones en la medida que sea difícil y costoso imitarlas; de lo contrario, apostarán por innovaciones incrementales (Katz y Shapiro, 1987).

\section{Novedad pura y novedad de mercado}

La novedad es la principal propuesta de valor de la innovación. No obstante, es necesario distinguir entre dos tipos de novedades para entender de mejor manera el fenómeno de la imitación: la novedad pura y la novedad de mercado.

\section{La novedad pura}

La novedad pura hace referencia a "algo" completamente nuevo. Es la definición que normalmente utilizan las oficinas de patentes de todo el mundo para determinar si sus solicitudes cumplen con el requisito de novedad. Esto quiere decir, que los productos o procesos, no deben encontrarse en el estado del arte y tampoco, en ninguna base de datos disponible al público al momento de presentar una solicitud de registro. No importa el idioma, el país o el mecanismo con el cual fue informado al público. La determinación de la novedad es un requisito sine qua non para obtener una patente de invención. Es un trabajo riguroso y requiere de peritos altamente calificados.

Como regla general, la mayor parte de las empresas que realizan innovación radical ingresan al mercado con novedades puras surgidas originalmente de la I+D. Éstas, utilizan portafolios de propiedad intelectual (PI) como barrera de entrada para disuadir a los imitadores, atraer a los inversionistas y/o licenciar su tecnología si es que no tienen las capacidades

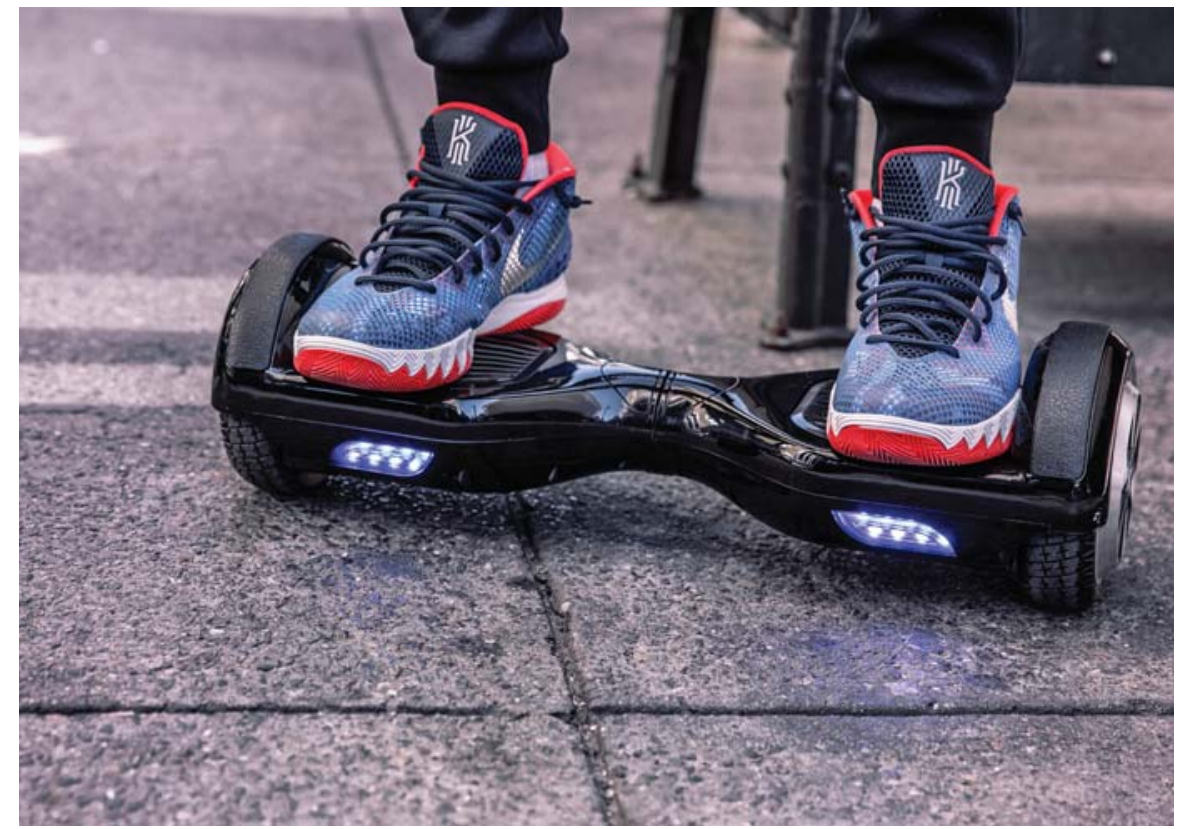

para explotar su propia innovación. Desde el punto de vista de la apropiabilidad, el gran activo de las novedades puras son las patentes de invención y los modelos de utilidad.

La determinación de la novedad pura se vuelve más compleja de admitir cuando las innovaciones no están acompañadas de consideraciones funcionales como ocurre con los diseños, dibujos y/o derechos de autor en general. Esto quiere decir que, para algunas invenciones, no es condición demostrar la aplicación industrial (el para qué sirve) a la hora de establecer una estrategia de apropiabilidad; lo que abre inevitablemente la posibilidad de "copiar" los diseños. 0 al menos, hacer pequeñas modificaciones en el modelo original para introducirlos en el mercado como un diseño propio.

\section{Novedad de mercado}

A diferencia de la novedad pura, en la novedad de mercado no interesa si el producto o servicio ya existe en el estado del arte o la técnica. Simplemente es desconocido para un grupo de usuarios en un rango de tiempo y/o lugar geográfico determinado. Seguramente, las bases que dieron origen a la novedad de mercado son ahora de dominio público, apareciendo en la actualidad reconfigurada o renovada. Otro atributo importante de la novedad de mercado es que el producto o servicio tiene que ser conocido - al menos en parte- por un grupo de usuarios para iniciar una tendencia y adopción de la nueva innovación. Aunque suene contradictorio, no existen novedades de mercado desconocidas. Las innovaciones tienen que alcanzar un umbral previo de adopción por parte de un grupo inicial de usuarios. Sin embargo, asimilar algo nuevo es un paso que no todos están dispuestos a dar. Es necesario disponer de los llamados ejecutores de tendencias y un grupo de seguidores para que las innovaciones se sostengan con la demanda del mercado (Vejlgaard, 2007). El proceso es un cruce que va desde la fase de diseño pre-paradigmático - la innovación se centra en el producto- hacia una fase de diseño paradigmático - la innovación centrada en el proceso-. Luego, una vez cruzada la frontera, la innovación se hace evidente y la novedad se desvanece paulatinamente perdiendo valor.

(3) Citado originalmente por Niosi, J. (2012, July) en Innovation and development through imitation (In praise of imitation). In meeting of the International Schumpeter Society, Brisbane, Australia (pp. 2-5). Igualmente, cuando me refiero a Schumpeter (1934). 
Desde el punto de vista de la apropiabilidad, el gran activo de las novedades de mercado son las marcas comerciales que ayudan a distinguir productos idénticos o similares de la competencia.

\section{La innovación imitativa}

Las imitaciones son principalmente innovaciones incrementales relacionadas directamente con las novedades introducidas por la competencia (Kleinknecht y col., 2002). Por ejemplo, el innovador genera nuevos productos, mientras que el imitador asimila rápidamente dichos productos dentro de firma, los adapta y los coloca a disposición del mercado.

Algunas características propias de los imitadores es que son significativamente más extrovertidos que las empresas innovadoras. Sus actividades están muy relacionadas con externalizar la I+D, son más activos en buscar cooperación y trabajan con equipos técnico-profesionales. En contras-

te, las empresas

innovadoras tienden a no confiar en la exploración del conocimiento externo: más bien, tienen un comportamiento introvertido y su personal es capital humano altamente especializado. Otro aspecto característico de los innovadores es que tienden a concentrarse en conocimiento específico, mientras que las empresas imitadoras buscan conocimiento externo inespecífico (Arvanitis \& Seliger, 2014).
Según Brondini (2012) los fenómenos de imitación van muy de la mano con el nuevo paradigma de la innovación abierta, que busca de alguna manera adquirir ideas, directrices, recursos y procesos desde el exterior para desarrollarlas internamente e implementar imitaciones que sean competitivas. En la actualidad, la imitación es un fenómeno real, está ocurriendo y tiene objetivos de rentabilidad a corto plazo y de bajo riesgo. Un ejemplo de éxito es la industria manufacturera de Taiwán. Allí, varias empresas han alcanzado un sofisticado manejo de sus recursos para hacer de la imitación una estrategia de innovación exitosa (Huang y col., 2010).

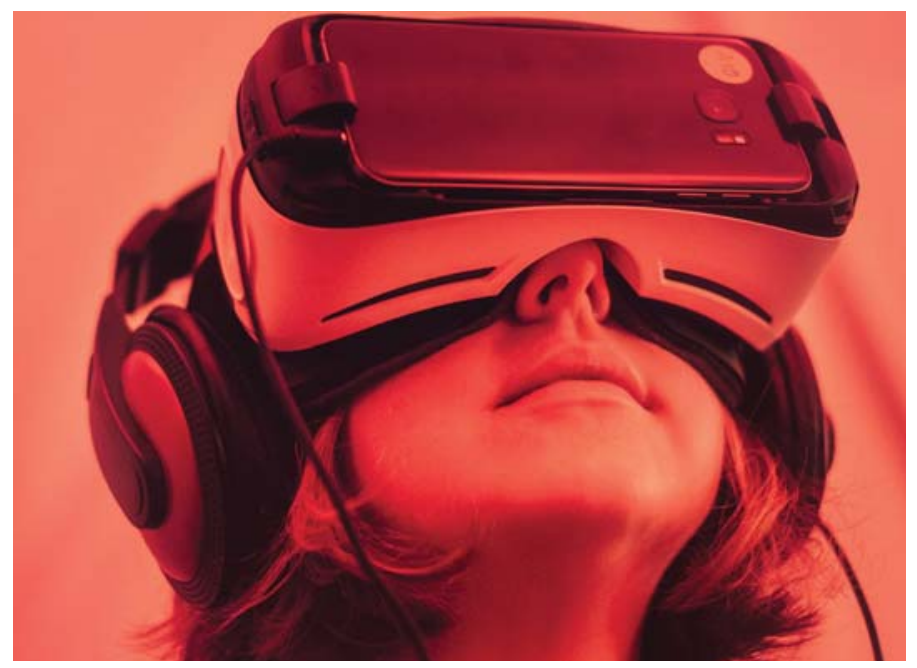

Es importante diferenciar que la imitación no implica necesariamente la violación de la propiedad intelectual como normalmente suele pensarse. Las regulaciones internacionales, los tratados de libre comercio y los propios acuerdos de colaboración tecnológica y de manufactura han transformado a la infracción de los derechos de PI en un negocio riesgoso y poco rentable.
En el caso de China, hubo una época entre la década del año 1950 y 1980, en donde la imitación pura era la principal forma de acceder a los mercados (Ding y col., 2011). Sin embargo, dicha situación cambió drásticamente después de la década del 90. En algunos sectores intensivos en $1+D$, las empresas chinas pasaron desde una fase imitativa hacia una actual fase de innovación independiente. Lo mismo parece haberse iniciado en la industria farmacéutica de la India (Duggan, 2016); lo que sugiere, que algunos fenómenos de imitación, evolucionan hacia un proceso de especialización y empujan a las empresas a refugiarse en PI propia. Lo anterior es muy positivo, pues se incentiva la exploración de nuevo conocimiento, se fomenta la cooperación y se fortalece la confianza en los mercados.

\section{Algunos tipos de imitación}

La imitación incluye varias maneras de adquirir la tecnología y reconfigurarla. Las más ineficientes y burdas de todas son ciertamente la piratería industrial, que incluye apropiarse de alguna manera de la propiedad intelectual ajena para lograr cierto grado de ventaja competitiva. Aunque se trate de diseños industriales legalmente distintos y levemente diferenciados, el hecho de parecerse al modelo original puede representar perjuicios económicos para los dueños de la tecnología o los líderes de la industria. También puede incitar al engaño, pues los clientes podrían pensar que están comprando el producto original $y$, sin embargo, reciben una réplica - a veces de mala calidad-del producto que esperaban. Este tipo de imitación es controversial pues, no siempre es fácil determinar la línea divisoria entre una copia exacta y la imitación. Normalmente los dueños del producto original se

\section{Cn la actualidad, la imitación es un fenómeno real, está ocurriendo y tiene objetivos de rentabilidad a corto plazo y de bajo riesgo)?}




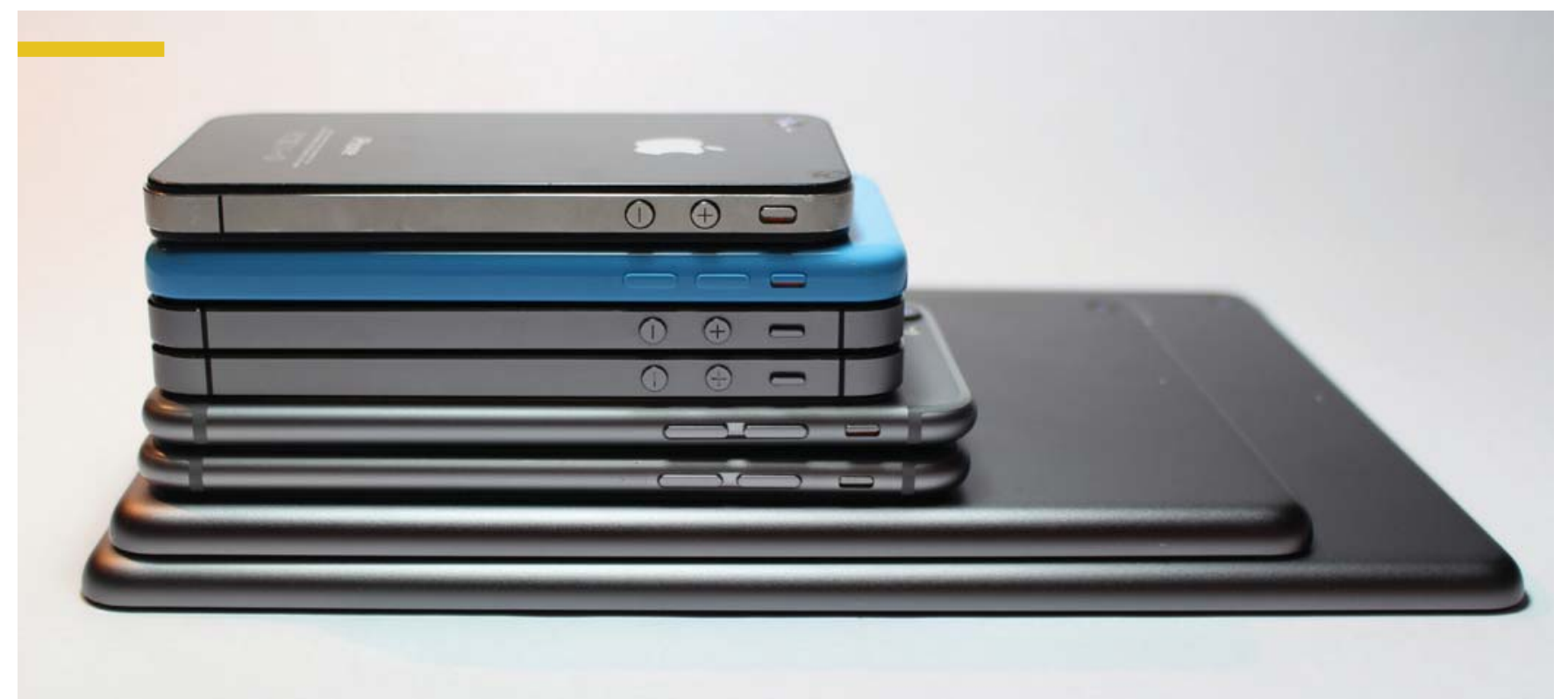

esfuerzan mucho por mantener cautivos a sus clientes, tratando de conectar su tecnología con servicios anexos como garantías o soporte técnico. Los formatos de imitación son variados y van desde clones (que involucra el diseño físico), ingeniería reversa (industria química y topología electrónica), emulación tecnológica (informática) y/o creación de análogos funcionales entre otras modalidades (Ulhoi, 2012). Este tipo de imitación se aprovecha de las marcas comerciales establecidas y también de la propiedad industrial "blanda" como son los dibujos, los diseños industriales, los circuitos integrados y algunos derechos de autor vinculados a la industria del software.

Afortunadamente existen mecanismos de innovación imitativa más sofisticados. Probablemente, uno de los promotores naturales de este hecho, ha sido la masiva adhesión de la industria tecnológica a los estándares de normalización y calidad. Esto ha permitido que muchas tecnologías de componente - parte de una arquitectura tecnológica mayor - puedan fabricarse bajo normas genéricas, lo que ha impulsado un verdadero mercado de tecnológicas complementarias o de sostén. Un ejemplo de ello es lo que ocurre con las cámaras réflex. Allí, la mayoría de los fabricantes diseñan su propuesta de valor centrados en el cuerpo del equipo fotográfico y no necesariamente en los "lentes" que tienen anillos de acoplamiento universales. Esto último ha permitido la emergencia de una verdadera industria de objetivos a nivel mundial ${ }^{4}$. Cualquier profesional que trabaja con ópticas de primer nivel, sabe que sus tecnologías de arquitectura no siempre cuentan con las mejores tecnologías de componente. Muchos prefieren ópticas genéricas, capaces de producir mejores resultados y a un bajo precio. La lección, es que los imitadores evolucionan hacia la especialización y sus innovaciones, - centradas mayoritariamente en tecnologías de componente- son capaces de satisfacer a mercados más sofisticados y exigentes.

Otro tipo de innovación imitativa es ahorrarse los esfuerzos de I+D para desarrollar productos derivados de patentes ya expiradas. Un ejemplo clásico es lo que ocurre en la industria de medicamentos genéricos. En una versión más elaborada, es posible crear nuevos agentes terapéuticos cambiando levemente sus principios activos para producir así, un resultado superior o similar al fármaco original (Niosi, 2017).
La adopción de estándares abiertos en la tecnología también promueve los procesos de imitación. En el caso del software, es común encontrarse con aplicaciones comerciales que utilizan código de fuente abierto para emular la interfaz, la usabilidad y hasta los diseños de la competencia (West \& Gallagher, 2006). Incluso, los líderes de la industria han comenzado a liberar su código-base con licencias menos restrictivas con el objeto de beneficiarse de la colaboración de sus usuarios en línea. Lo mismo parece estar replicándose en la industria del hardware en donde varias escuelas de ingeniería de prestigiosas universidades del mundo, han comenzado a utilizar plataformas de desarrollo abiertas en sus procesos de formación ${ }^{5}$. El objetivo de estas iniciativas es difundir, democratizar y liberar la tecnología de cualquier derecho de exclusividad para beneficio de la sociedad en su conjunto ${ }^{6}$.

\section{Conclusión}

Las innovaciones surgidas de la I+D son claves para el desarrollo de las economías. Sin embargo, este imperativo estratégico no parece ser el camino obvio para la mayoría de los países emergentes de la región. En el caso de Chile, la baja inversión

(4) Recomiendo la última revisión de Jim Fisher en la PC Mag. The Best DSLR Lenses of 2017 disponible en https://www.pcmag.com/article2/0,2817,2421983,00.asp (5) Arduino es una compañía de hardware libre. Una comunidad tecnológica que diseña y manufactura placas de computadora. En Chile varias escuelas de ingeniería utilizan Arduino como base para enseñar electrónica y crear tecnología. Para saber más visitar https://www.ing.uc.cl/ingenieria-uc-lanza-curso-gratuito-online-para-aprender-crear-robots-caseros/ (6) Libre de derechos de exclusividad quiere decir que son tecnologías de dominio público. Cualquiera puede apropiarse de ellas, modificar el código y generar tecnologías derivadas.

(7) En Índice Global de Innovación mide la creación de tecnología, conocimiento y creatividad (outputs) principalmente mediante indicadores de propiedad industrial y derechos de autor entre los que destacan las patentes, modelos de utilidad, marcas, licencias, contratos de I+D, $n^{\circ}$ de artículos publicados y citados entre otros. Para saber más visitar http://www.wipo.int/publications/en/details.jsp?id=4193 


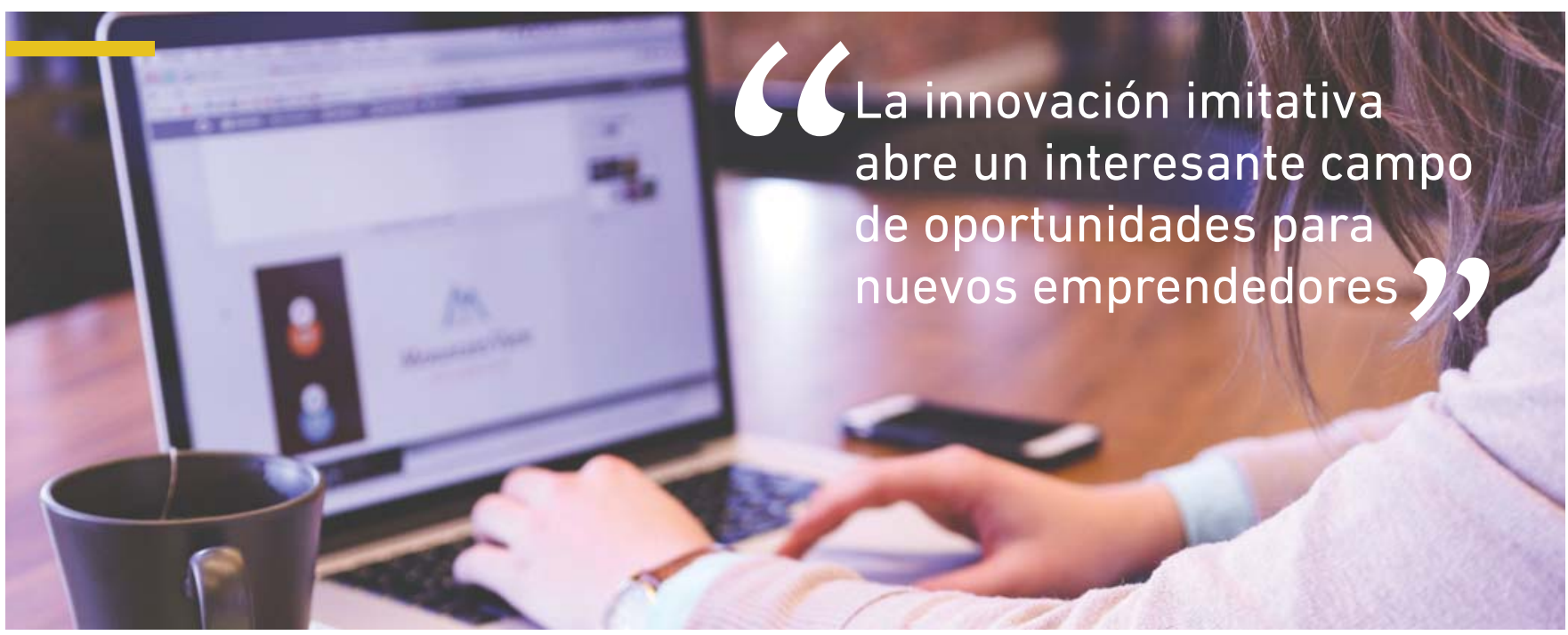

en I+D por parte de las empresas, la poca vinculación de las universidades con el sector productivo y el incipiente compromiso del gobierno con la ciencia, hacen improbable el surgimiento de novedades puras capaces de empujar la creación de nuevos negocios de base científico- tecnológica (Jiménez, 2016).

Una alternativa menos tradicional frente a la falta de recursos es la imitación. 0 sea, especializarse estratégicamente en innovaciones incrementales, ahorrándose la I+D y sacrificando así, la posibilidad de establecer barreras de entrada mediante la propiedad intelectual. Innovar frecuentemente es caro y riesgoso. La mayoría de las veces los proyectos no alcanzan a llegar al mercado, quedan inconclusos y los fracasos son escasamente documentados por los journals académicos. También ocurre algo de lo que pocos hablan: muchas empresas nuevas y de rápido crecimiento son imitadas y/o luego absorbidas por compañías establecidas; lo que sugiere que los fenómenos de imitación no solamente van desde los "chicos" hacia los "grandes", también ocurren de manera inversa.

Hoy en día, los resultados de la innovación se miden principalmente en activos de propiedad intelectual que generan valor y favorecen la atracción de inversiones. No obstante, este foco estratégico no es compartido por los imitadores que prefieren adaptar, adquirir o implementar modelos desde el exterior en vez de realizar I+D propia. En el mejor de los casos, prefieren externalizar sus necesidades de investigación. Se trata de empresas exitosas y no ven en la PI una fuente de ventajas competitivas. Más bien, prefieren hacer alianzas, ser flexibles, adquirir activos del estado del arte y reconfigurarse rápidamente según los intereses del mercado.

Por el momento, la imitación no parece tener mucho sustento en la literatura como una alternativa efectiva al desarrollo económico. Sin embargo, también sería imprudente descartar su impacto en los mercados globales y/o economías emergentes. Al parecer, se trata de un fenómeno poco estudiado, intensamente dinámico y cuya evidencia no está reflejada en los actuales índices de innovación y competitividad. Lo cierto es que la "innovación imitativa" sí está ocurriendo y podría abrir un interesante campo de oportunidades para nuevos emprendedores.

\section{Referencias}

Arvanitis, S., \& Seliger, F. (2014). Imitation versus Innovation: What Makes the Difference?

Brondoni, S. M. (2012). Innovation and Imitation: Corporate Strategies for Global Competition. Symphonya, (1), 10.

Christensen, C. (2013). The innovator's dilemma: when new technologies cause great firms to fail. Harvard Business Review Press.

Coad, A. (2011). Appropriate business strategy for leaders and laggards. Industrial and Corporate Change, 20(4), 1049-1079.

Ding, J., Xue, Y., Liang, H., Shao, R., \& Chen, Y. (2011). From imitation to innovation: A study of China's drug R\&D and relevant national policies. Journal of technology management \& innovation, 6(2), 1-13.
Huang, J. Y. Chou, T. C., \& Lee, G. G. (2010). Imitative innovation strategies: Understanding resource management of competent followers. Management Decision, 48(6), 952-975.

Jiménez, A. (2016). Relaciones universidad-empresa: Hacia una productividad basada en innovación. Gestión y Tendencias, 1(2), 7-10.

Katz, M. L., \& Shapiro, C. (1987). R and $D$ rivalry with licensing or imitation. The American Economic Review, 402-420.

Kleinknecht, A., Van Montfort, K., \& Brouwer, E. (2002). The nontrivial choice between innovation indicators. Economics of Innovation and new technology, 11(2), 109121.
Niosi, J. (2012, July). Innovation and development through imitation (In praise of imitation). In meeting of the International Schumpeter Society, Brisbane, Australia (pp. 2-5).

Niosi, J. (2017). Imitation and innovation new biologics, biosimilars and biobetters. Technology Analysis \& Strategic Management, 29(3), 251-262.

March, J. G. (1994). The evolution of evolution. Evolutionary dynamics of organizations, 39-49.

Duggan, M., Garthwaite, C., \& Goyal, A. (2016). The Market Impacts of Pharmaceutical Product Patents in Developing Countries: Evidence from India. American Economic Review. 106(1), 99-135
Schumpeter, J. A. (1934). The theory of economic development. Cambridge. MA: Harvard.

Ulhøi, J. P. (2012). Modes and orders of market entry: revisiting innovation and imitation strategies. Technology Analysis \& Strategic Management, 24(1), 37-50.

Vejlgaard, H. (2007). Anatomy of a Trend. McGraw Hill Professional.

Vélez Montoya, A. (2007). Homo Sapiens.

West, J., \& Gallagher, S. (2006). Challenges of open innovation: the paradox of firm investment in opensource software. R\&d Management, 36(3), 319-331. 